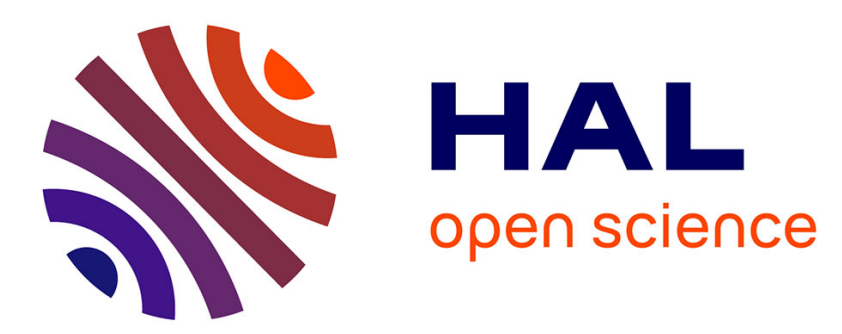

\title{
Continuité et innovation littéraire en Angleterre au XIIe siècle: la prédication de la militia Christ
}

Stephen Morrison

\section{To cite this version:}

Stephen Morrison. Continuité et innovation littéraire en Angleterre au XIIe siècle: la prédication de la militia Christ. Cahiers de Civilisation Médiévale, 2001, 44 (174), pp.139-157. 10.3406/ccmed.2001.2798 . halshs-01338240

\section{HAL Id: halshs-01338240 \\ https://shs.hal.science/halshs-01338240}

Submitted on 28 Jun 2016

HAL is a multi-disciplinary open access archive for the deposit and dissemination of scientific research documents, whether they are published or not. The documents may come from teaching and research institutions in France or abroad, or from public or private research centers.
L'archive ouverte pluridisciplinaire HAL, est destinée au dépôt et à la diffusion de documents scientifiques de niveau recherche, publiés ou non, émanant des établissements d'enseignement et de recherche français ou étrangers, des laboratoires publics ou privés. 


\section{Continuité et innovation littéraire en Angleterre au XIle siècle : la} prédication de la militia Christ

\section{Stephen Morrison}

\section{Citer ce document / Cite this document :}

Morrison Stephen. Continuité et innovation littéraire en Angleterre au XIle siècle : la prédication de la militia Christ. In: Cahiers de civilisation médiévale, 44e année (n¹74), Avril-juin 2001. pp. 139-157;

doi : 10.3406/ccmed.2001.2798

http://www.persee.fr/doc/ccmed_0007-9731_2001_num_44_174_2798

Document généré le 01/06/2016 


\begin{abstract}
English literary historians of the twelfth and thirteenth centuries have for long sought evidence for the continuity of the Old English literary tradition in the texts known collectively as the Katherine Group and in related texts. They have further identified the south-west of the country as the hub of this persistent interest in vernacular activity. However, detailed consideration of one important but neglected mid-tolate twelfth-century text, the Ormulum, suggests that this picture is imbalanced. By comparing the verbal articulation of the Militia Christi, a topos central to the work of Orm and to abbot /Elfric, the most prolific of late Old English homilists and the one whose works were carefully preserved by successive generations after his death, I attempt to show that the estimate of the strength of the older vernacular tradition has been overstated. It is also part of my purpose to suggest that literary activity in the southeast of the country was as vigorous as that claimed for the south-west.
\end{abstract}

\title{
Résumé
}

De façon générale, la critique littéraire de l'Angleterre des XIle et XIIle s. tend à souligner la position clef qu'occupent les textes connus sous le nom de "Katherine Group » dans la discussion de la survie de la tradition littéraire vieil-anglaise. De plus, elle associe cette prédilection pour la composition en langue vernaculaire avec le sud-ouest du pays, aux dépens d'autres régions. Cependant, le témoignage de l'Ormulum, un texte quelque peu négligé de la deuxième moitié du Xlle s., laisse penser que cette prise de position manque d'équilibre. A partir d'une comparaison de la présentation d'un topos - celui de la Militia Christi - central à l'Ormulum et à l'œuvre de l'abbé $F$ Elfric, le plus important des sermonnaires vieil-anglais, dont le programme d'instruction fut conservé par des générations successives après sa mort -, je m'efforce de démontrer que cette notion de " survie » est susceptible d'une interprétation différente. En même temps, je suggère que l'activité littéraire traditionnellement associée au sud-ouest du pays a fait également partie de la vie intellectuelle de la région du sud-est de l'Angleterre. 


\title{
Stephen MORRISON
}

\section{Continuité et innovation littéraire en Angleterre au XII ${ }^{\mathrm{e}}$ siècle : la prédication de la militia Christi}

Christi miles stm : pugnare mihi non licet ${ }^{1}$

\begin{abstract}
RÉSL'MÉ:
De façon générale, la critique littéraire de l'Angleterre des XII" et XIII' s. tend à souligner la position clef qu'occupent les textes connus sous le nom de "Katherine (iroup » dans la discussion de la survie de la tradition littéraire vieil-anglaise. De plus, elle associe cette prédilection pour la composition en langue vernaculaire avec le sud-ouest du pays, aux dépens d’autres régions. Cependant. le témoignage de l'Ormulum, un texte quelque peu négligé de la deuxième moitié du xıß s. laisse penser que cette prise de position manque d'équilibre. A partir d'une comparaison de la présentation d'un topos - celui de la Militia Christi - central à l'Ormulum et à l'œuvre de l'abbé Ælfric. le plus important des sermonnaires vieil-anglais. dont le programme d'instruction fut conservé par des générations successives après sa mort -. je m’efforce de démontrer que cette notion de "survie » est susceptible d'une interprétation différente. En même temps, je suggère que l'activité littéraire traditionnellement associéc au sud-oucst du pays a fait également partie de la vie intellectuelle de la région du sud-est de l'Angleterre.
\end{abstract}

\section{ABSTRACT}

English literary historians of the twelfth and thirteenth centuries have for long sought evidence for the continuity of the Old English literary tradition in the texts known collectively as the Katherine (jroup and in related texts. They have further identified the south-west of the country as the hub of this persistent interest in vernacular activity. However, detailed consideration of one important but neglected mid-to-late twelfth-century text, the Ormulum, suggests that this picture is imbalanced. By comparing the verbal articulation of the Militia Christi, a topos central to the work of Orm and to abbot Ælfric. the most prolific of late Old English homilists and the one whose works were carefully preserved by successive generations after his death. I attempt to show that the estimate of the strength of the older vernacular tradition has been overstated. It is also part of my purpose to suggest that literary activity in the south-east of the country was as vigorous as that claimed for the south-west.

\section{Le problème}

Suite aux bouleversements politiques et militaires qui ont marqué la première moitié du $\mathrm{XI}^{\mathrm{C}} \mathrm{S}$. l'Angleterre est entrée dans une période de mutations rapides: la mise en place progressive des institutions normandes, l'introduction du français comme deuxième langue vernaculaire (dont l'usage s'est limité aux milieux animés par les conquérants). et la naissance d’une littérature 
anglo-normande en témoignent ${ }^{2}$. Dans le contexte de ce nouveau climat, les spécialistes littéraires ont tenté à plusieurs reprises de répondre à une question dont l'importance s'est fait sentir d'emblée: dans cette période transitoire, les traditions de composition en langue vernaculaire sont-elles, en entier ou en partie, maintenues et, si oui, jusqu'à quelle date approximative, ct dans quelle(s) rćgion(s) du pays?

La réponse jusqu'ici unanime à ces questions veut que ce soient les compositions en prose du $\mathrm{XII}^{\mathrm{e}}$ s. d'une part, et celles des débuts du XIII' s., d'autre part, qui constituent la preuve la plus éloquente de continuité, et que les régions du pays les plus concernées par cette activité littéraire, à tendance conservatrice, sont celles des Midlands occidentales et du Sud-Ouest. Dans ces régions, les scriptoria de Worcester, de Wigmore et de Hereford, notamment, auraient joué un rôle décisif ${ }^{3}$.

Les textes du XII $\mathrm{e}$. sur lesquels se fonde cet argument sont constitués principalement des sermons anglais de l'abbé Ælfric (env. 945 - env. 1015), en particulier ses Sermones Catholici (ciaprès $S C$ ), publiés en deux séries, et ses Vies des Saints $(V S)$, et aussi de ceux de Wulfstan, évêque de Worcester et archevêque de York $(\dagger 1023)^{4}$. Ælfric fut d'abord moine de Cerne (dans le Devon), puis, de 995 à 1015 environ, (date présumée de sa mort), abbé d'Eynsham (Oxfordshire). Il fut l'élève d'Æthelwold à Winchester et, avec son contemporain Wulfstan, il faisait partie de la première génération de moines qui bénéficiaient du mouvement de réforme bénédictine amorcée vers le milieu du $\mathrm{x}^{\mathrm{e}} \mathrm{s}$.

Le personnage d'Ælfric ainsi que ses réussites littéraire sont connus. Comme l'avait fait Bède le vénérable (env. 673-735) au VIII' s., il a transmis à son public tout un programme d'enseignement religieux. Si l'importance de Bède se trouve dans sa capacité à transmettre la culture patristique (notamment la pensée d'Augustin et de Grégoire le Grand) en latin, c'est dans la constitution d'un ensemble cohérent et orthodoxe de sermons en anglais, reflétant cette tradition patristique, que réside celle de son successeur ${ }^{5}$. Les Sermones Catholici, son ouvre majeure, ont été publiés entre 990 et 994 environ. Chaque série, comme nous informe Ælfric lui-même dans la préface anglaise de la deuxième série ${ }^{6}$, comporte une quarantaine de textes destinés à être prêchés tous les deux ans. Ses modèles immédiats sont carolingiens ${ }^{7}$. Les quatre-vingts sermons sont organisés

2. Sur ces points, voir Marjorie ChibNAl..., Anglo-Norman England, 1066-1166. Oxford, 1986: - Ian SHORT, "On Bilingualism in Anglo-Norman England". Romance Philology. 33. 1980. p. 467-479: - M. Dominica LEGGE, AngloNorman Literature and its Background. Oxford, 1963. Notons également l'importante étude récente de William CALIN. The French Tradition and the Literature of Medieval England. Toronto, 1994.

3. Toujours utiles sur cette question sont les contributions de R.M. WILSON, Early Middle English Literature. Londres. 1939. ch. V. et l'introduction de son éd. de Sawles Warde. Kendal, 1938 (Leeds School of English Language Texts and Monographs. III).

4. Il est évident que le genre littéraire le plus pertinemment évoqué dans ce contexte multilingue est le sermon en langue vernaculaire. destiné à un public peu ou pas instruit. R.W. Chambers, dans un essai qui est vite devenu un classique du genre, a fait remarquer que "Great bishops and abbots, although they might speak Latin among themselves. and French to their secular peers. had to consider the souls of those who could speak neither ". Voir son On the Continuity of English Prose from Alfred to More and his School, Londres, 1932 (Early English Text Society (ci-après EETS), os 191A). p. XCIII-XCIV. Les éditions de ces textes utilisées dans cette étude sont les suivantes: Peter CLFMOFs, Elfric's Catholic Homilies: the First Series, Texts, Oxford. 1997 (EETS ss 17) : - Malcolm GodDEN, Alfric's Catholic Homilies: the Second Series, Texts, Londres, 1979 (EETS ss 5) ; - Walter W. Skrat, AElfric's Lives of Saints, réimpr. de l'éd. 1881-1900. Londres/New York. 1966 (EETS os 76. 82, 94 et 114). Dans la préface latine de la première série des $S C$, Elfric indique à quel genre litteraire appartient, selon lui, son ceuvre: "Scilicet sancte scripture in nostram consuetam sermocinationem ob edificationem simplicium qui hanc notunt tantummodo locutionem" (Cl.1:MOL:S, éd. cit. p. 173). En dépit du fait que le sermo et la homilia sont des compositions distinctes en méthode et en structure. et que tous les deux sont représentés dans l’œuvre d’Ælfric, je retiendrai le terme "sermon" dans la présente discussion par souci de commodité.

5. Le nom de Bède figure parmi les aluctoritates mentionnées par Ælfric dans la préface latine de la première série des SC (Cl.EmOEs. éd. cit., 1997, p. 173).

6. GoDDIN. éd. cit., p. 3.

7. La discussion la plus récente. avec un résumé des résultats de plusieurs décennies de recherche sur la question des sources. et une bibliographie très importante. est celle de Joyce HILL. "Translating the Tradition: Manuscripts, Models and Methodologies in the Composition of Elfric's Catholic Homilies". Bulletin of the John Rylands University Library of Manchester. 79. 1997. p. 43-65. 
selon l'année liturgique, en proposant les discours pour certains dimanches et fêtes non dominicales principales de la vie du Christ. Mais il est à remarquer que, parmi eux. non loin de la moitié ont été conçus pour les fêtes des saintes et celles de la Vierge. S'ajoutent à ses sermons deux séries supplémentaires. les Vies des Saints, où sont honorés ceux qui ne figurent pas dans les SC. Avec sa Grammaire, ainsi que son De Temporibus Anni, Ælfric crée un programme d'instruction complet, rigoureusement orthodoxe (on se souvient de son souci de faire disparaître le micel gedwyld qu'il avait rencontré dans d'autres livres anglais, non identifiés). et bien pratique : l'érudition des auteurs patristiques et carolingiens est désormais à la portée de ceux qui ne connaissent pas le latin ${ }^{8}$.

L'importance suprême d’Ælfric dans le débat sur la survie des traditions anglaises littéraires après la Conquête a été soulignée à plusieurs reprises. Fernand Mossé. par exemple, remarque que : "L'influence de ses auvres, recopiées, imitées, remaniées jusqu'au XII" s. a été considérable: après la conquête elles ont largement contribué à maintenir vivante la prose religieuse anglaise et, sans doute, assurer sa continuité ". Plus d'un demi-siècle après Mossé, Theodore Leinbaugh a écrit : "[t]he success of Ælfric's educational mission can be partially measured by the large number of surviving Anglo-Saxon manuscripts that preserve his writings: these manuscripts bear witness to the esteem of his contemporaries and to those scribes and scholars who continued to copy his writings for the next 150 years " ${ }^{10}$. Dans une perspective plus large, André Crépin et Hélène Taurinya Dauby ont récemment résumé de manière claire les grandes lignes de cette prise de position critique :

Non seulement on recopia fidèlement les collections anciennes [de sermons], mais on les modernisa. Ou bien on traduisit en anglais des sermons latins, par exemple de Maurice de Sully, évêque de Paris (1160-96). Importantes, non pas pour leurs qualités esthétiques mais pour leur rôle de maillons. sont les œuvres des Midlands occidentales. La région avait été moins soumise à l'influence continentale que le Sud et l'Est de l'Angleterre, et n’avait pas subi de dévastation comme le Nord, deux fois ravagé, d'abord par les Scandinaves puis par Guillaume le Conquérant. En outre, la région avait bénéficié du long épiscopat de Wulfstan. évêque de Worcester de 1062 à 1095 [à ne pas confondre avec Wulfstan. son prédécesseur, contemporain d’Ælfric. mentionné plus haut]. qui favorisa la tradition vicil-anglaise. Cette tradition, attestée par une Vie en vicil-anglais (IX ${ }^{\mathfrak{E}}$ siècle) de Saint Chad, évangélisateur de la Mercie, et peut-être par des sermons appelés Lambeth Homilies, forme un jalon entre littérature homilétique vicil-anglaise et le groupe moyen-anglais de la Vic de sainte Catherine ${ }^{11}$.

La mention ici du groupe de textes portant le nom de sainte Catherine m'amène à élargir cette discussion. en prenant en considération la question de la place occupée par les compositions en prose des débuts du XIII ${ }^{\circ}$ s. évoquées au début de cet article. Car, si les Lambeth Homilies et autres collections du XII' s. maintiennent (comme nous le verrons plus tard) l'essentiel de l'enseignement ælfricien, les textes du groupe de la Vie de sainte Catherine ouvrent de nouvelles perspectives. Ces derniers, étroitement liés en date et en provenance au traité appelé Ancrene Wisse (Guide pour Recluses), sont au nombre de cinq : d'abord. un traité sur la virginité. Hali Meiðhad (c'est-à-dire "la virginité consacrée»), une homélie allégorique, Sawles Warde («la

8. Flfric pose le problème de la fiabilité des livres anglais dans la préface anglaise de la première série des $S C^{\circ}$ (Clemols. éd. cit. p. 174). Pendant toute sa vie. il lutta contre les propos hérétiques ou apocryphes. Un deuxième exemple est fourni par son refus de s'associer aux gedwolmen qui racontaient, sans doute d'une manière fantaisiste. l'histoire de la naissance de Marie. Voir l'introduction de son Nativitas Sanctae Mariae Virginis, dans Bruno Assidaxi. éd.. Angelsächsische Homilien und Heiligenleben. réimpr. avec introd. de P. (I.teMotss de l'éd. de 1889. Darmstadt. 1964 (Bibliothek der angelsächsischen Prosa. III). p. 24. lignes 5 à 9. Le va. gedwyld veut dire "erreur". "hérésie": les gedwolmen sont ceux qui la répandent.

9. Fernand Mossi., Manuel de langlais du moyen âge des origines an xne siécle. I : Vieil anglais. Paris, 1945. 2 vol.. t. 1. p. $245-246$.

10. Voir son article sur ÆElfric. dans Paul E. Szarmach et al. éd.. Medieral England: an Encyclopedia. New York/Londres. 1998. p. 4-7.

11. André CRépIn et Hélène Tatrinya Dat:By. Histoire de la littérature anglaise du moyen âge. Paris. 1993. p. 61. Sur l'importance de la vie de saint (had. voir l’éd. de Rudolf VItriskrt:Yre. The Life of Saint Chad: an Old linglish Homily. Amsterdam. 1953. Sur les Lambeth Homilies. voir infra. 
garde de l'âme "), ainsi que les Vies de trois vierges martyres: Julienne, Marguerite et Catherine (d'où le nom du groupe). Bien qu'ils donnent une nouvelle impulsion à la tradition de composition en langue anglaise, ces textes du Sud-Ouest ont souvent été considérés comme les successeurs naturels de ceux qui constituent le corpus homilétique vieil-anglais ${ }^{12}$.

Ainsi, pour bon nombre de chercheurs, bien que le maintien de la tradition de composition en anglais ne soit pas exclusivement une affaire de langue, c'est l'emploi ininterrompu de l'anglais qui constitue un critère essentiel à la formulation de cette notion de continuité. Cependant, la prise en compte d'autres critères apporte des nuances à cette affirmation. Sur le plan stylistique, par exemple, il existe des liens indéniables entre les Vies des Saints d'Ælfric et celles du groupe de la Vie de sainte Catherine : il est bien connu que ces dernières s'inspirent de son œuvre dans l'utilisation (parfois excessive, attribuable à un manque de discernement), d'une prose rythmée, fondée sur le principe de l'allitération ${ }^{13}$. Des études plus récentes ont démontré cependant l'influence des modèles latins dans la constitution des stratégies rhétoriques des auteurs anglais, indiquant ainsi une certaine indépendance du modèle indigène. Quant au contenu théologique et intellectuel, leur dette envers le corpus vieil-anglais n'est que partielle, et il semblerait que, dans ces domaines aussi, ils affichent une affinité avec la renaissance intellectuelle de l'époque, malgré la préférence de leurs auteurs pour l'anglais comme langue de rédaction ${ }^{14}$.

Il existe donc une continuité linguistique, certes; la continuité de l'instruction religieuse aux laïques est également assurée. Mais l'argument, tel qu'il est présenté ci-dessus, laisse sans réponse plusieurs questions importantes. Par exemple, quel est le caractère précis des copies de ces textes anciens réalisées pendant ce temps? Que peut-on déduire de leur témoignage? L'activité littéraire associée aux auteurs du Sud-Ouest du pays est-elle entièrement représentative du rôle joué par l'anglais pendant cette période? Il me semble que l'argument avancé jusqu'ici est le fruit d'une analyse incomplète des preuves manuscrites à notre disposition. De plus, des considérations proprement littéraires - orientation et articulation des topoi hérités de la tradition latine, mise en forme en anglais de ces idées reçues, phénomène du choix lexical qui donne à une image littéraire quelconque une identité linguistique spécifique - ne semblent pas avoir trouvé leur place dans cette discussion.

Il n'est nullement dans mes intentions de jeter le doute sur les grands traits de cet argument; je me propose plutôt de mieux le définir. Pour ce faire, j'adopterai une approche double. Dans un premier temps, j'examinerai les manuscrits du XII $\mathrm{e}$. qui conservent des textes en vieil-anglais du point de vue de leur provenance (lorsque celle-ci est connue), leur date et leur contenu, afin de mieux comprendre le phénomène de reproduction continue de textes vieil-anglais à travers ce siècle. Dans un deuxième temps, j'attirerai l'attention sur une collection d'homélies en vers dont le témoignage n'a jamais été pris en considération dans la discussion de continuité littéraire. S'agissant d'homélies exégétiques pour les dimanches et les fêtes majeures non dominicales, rédigées par un chanoine augustinien, Orm, cette collection offre l'occasion de comparer les stratégies littéraires d'un innovateur en matière de prédication avec celles de ses illustres prédécesseurs. Au cœur de la conception de la vie religieuse prêchée par Orm et par les auteurs vieil-anglais réside la métaphore de la militia Christi, la lutte spirituelle contre les forces du mal.

12. Pour John Burrow, p. ex., "The remarkable school of anglo-saxon prose writers, of whom the best known are Elfric (c. 955 - 1020) and Wulfstan (d. 1023). found successors two hundred years later in the authors of the Ancrene Wisse and the homilies of the Katherine Group...". Voir son Medieval Writers and their Work: Middle English Literature and its Background, 1150-1500. Oxford. 1982. p. 4.

13. Voir l'étude de Dorothy Bethurum, "The Connection of the Katherine Group with Old English Prose ". Journal of English and Germanic Philology, 34, 1935. p. 553-564.

14. Pour une édition récente (de morceaux sélectionnés) avec une discussion importante, voir Bella MitLLi: et Jocelyn Wogan-Browne. Selections from the Katherine Group and Ancrene Wisse. Oxford, 1990. Également Bella Milult. "Hali Meiohad". 'Sawles Warde'. and the Continuity of English Prose." dans E.G. Staniry et Douglas Gray. éd.. Five Hundred Years of Words and Sounds : A Festschrifi for Eric DOBSON, Woodbridge. 1983, p. 100-108. Sur le Guide pour recluses, voir E.J. Dobson. The Origins of Ancrene Wisse. Oxford. 1970) et A.S.G. Edwards, éd., Middle English Prose : A Critical Ginide to Major Authors and Genres. New Brunswick. 1985, ch. 1. 
C'est cette observation qui a déterminé mon choix thématique (annoncé dans le titre de cet article), et qui me permettra de tenter de formuler une juste appréciation de la place de l'anglais littéraire à la charnière de deux époques.

\section{Les preuves manuscrites}

Passons d'abord aux productions littéraires anglaises témoignées par les manuscrits écrits entre 1100 et 1200 environ. Bien qu'il faille reconnaître le caractère souvent fortuit de la survie d'un manuscrit médiéval jusqu'à nos jours, l'ensemble des manuscrits vieil-anglais présentés ci-dessous possèdent des traits très cohérents, laissant penser qu'ils reflètent assez fidèlement les préoccupations des scriptoria de leur temps. Neil Ker, dans son Catalogue of Manuscripts Containing Anglo-Saxon ${ }^{15}$, outil indispensable à tout chercheur dans ce domaine, dresse une liste de vingt-sept manuscrits appartenant à ce siècle. Je rajoute aux renseignements fournis par Ker des indications de contenu pour chaque manuscrit concerné. La liste se lit comme suit ${ }^{16}$ :

1. [Ker \$18] Cambridge, University Library Ii.1.33. XII2. Provenance inconnue. Homéliaire. Essentiellement Ælfric : SC I, SC II, VS et Hexameron. Textes remaniés au XIII' s. ; maximes en vers rajuulées.

2. [Ker \$56] Cambridge, Corpus Christi College 302. XI/XII. Provenance inconnue. Homéliairc. Essentiellement Ælfric : SC I, SC II.

3. [Ker §57] Cambridge, Corpus Christi College 303. XII1. Provenance inconnue, Rochester? Homéliaire. Essentiellement Ælfric : SC I, SC II.

4. [Ker \$62] Cambridge, Corpus Christi College 367, première partie. XII2. Provenance inconnue. Essentiellement Ælfric : De Temporibus.

5. [Ker \$63] Cambridge, Corpus Christi College 367, deuxième partie. XII. Provenance inconnue. Essentiellement Ælfric : SC I.

6. [Ker §65] Cambridge, Corpus Christi College 383. XI/XII. Londres, St. Paul's.

Lois des rois Cnut, Edward et Edmund.

7. [Ker \$89] Cambridge, Trinity College R.9.17. XI/XII. Provenance inconnue.

Ælfric, Grammaire; Caton.

8. [Ker \$91] Cambridge, Trinity College R.17.1. XIImed. Canterbury.

Psautier de Canterbury, dit d'Eadwine. Psautier trilingue.

9. [Ker \$139b] Londres, British Library, Cotton Caligula A. xv. fol. 142ff. XI/XII. Provenance inconnue. Ælfric, De Temporibus Anni (extraits).

10. [Ker \$148] Londres, British Library, Cotton Domitian viii. XI/XII. Canterbury, Christ Church. Chronique anglo-saxonne bilingue (s'achève sub anno 1058).

11. [Ker \$150] Londres, British Library, Cotton Domitian ix. XII. Provenance inconnue. Chronique anglo-saxonne (fragment)

12. [Ker \$153] Londres, British Library, Cotton Faustina A.ix. XII1. Provenance inconnue. Homéliaire. Flfric : SC I, SC II et quelques homélies de Wulfstan. Textes remaniés au XII $\mathrm{s}$. (Lié textuellement aux mss Cambridge, University Library Ii.4.6. XImed et Cambridge. Corpus Christi College 302, $\mathrm{XI} / \mathrm{XII})$.

13. [Ker $§ 154 b]$ Londres, British Library. Cotton Faustina A.x. XII' ${ }^{1}$. Provenance inconnue. Regula Benedicti.

14. [Ker \$159] Londres, British Library, Cotton Julius A.ii. fol. 136-44. XIImed. Provenance inconnue. Adrian and Ritheus (dialogue): Caton.

15. Oxford. 1957, avec "A Supplement to Catalogue of Mamuscripts Containing Anglo-Saxon", Anglo-Saxon England. 5. 1976. p. $121-131$.

16. Les renseignements donnés pour chaque manuscrit sont : numéro de catalogue donné par Ker. lieu de conservation actuel, date, provenance du manuscrit (lorsque celle-ci est connue) et contenu. Le système de datation adopté par Ker est expliqué dans le Catalogue. p. xx. 
15. [Ker \$173] Londres, British Library, Cotton Otho A.xiii. XII. Détruit.

Homélies.

16. [Ker \$209] Londres, British Library, Cotton Vespasian D.xiv. XIImed. Rochester/Canterbury. Homéliaire. Essentiellement ÆElfric SC I, SC II avec deux morceaux traduits après la Conquête (Honorius d'Autun et Raoul d'Escures).

17. [Ker \$215] Londres, British Library, Cotton Vitellius A. Xv, première partic. XIImed. Southwick (Hampshire) au XIII ${ }^{e}$ s. Augustin, Soliloquiae, Évangile de Nicodème, Salomon et Saturne.

18. [Ker \$226] Londres, British Library, Harley 55. XIImed. Provenance inconnue. Lois du roi Cnut.

19. [Ker \$245] Londres, British Library, Royal 1.A.xiv. XII2. Canterbury.

Évangiles. Texte étudié et glosé aux XIII' et XIVe s.

20. [Ker \$305] Oxford, Bodleian Library, Bodley 180. XII1. Provenance inconnue. Boèce, De Consolatione Philosophiae.

21. [Ker \$310] Oxford, Bodleian Library, Bodley 343. XII2. Midlands occidentales.

Homéliaire. Essentiellement Ælfric, SC I, SC II : Homélies de Wulfstan, le poème intitulé "The Grave » [La Tombe].

22. [Ker \$325] Oxford. Bodleian Library, Hatton 38. XII/XIII. Canterbury.

Évangiles. Copie de Londres. British Library, Royal 1.A.xiv ( $\mathrm{N}^{\circ} 19$ supra), ou tirée du même modèle.

23. [Ker \$333] Oxford, Bodleian Library, Hatton 116. XII1. Midlands occidentales, Worcester? Homéliaire. Essentiellement Ælfric SC I. Quelques textes de Wulfstan. Ms. glosé par la tremulous hand (XIII $\left.^{\text {S. }}\right)^{17}$.

24. [Ker \$346] Oxford, Bodleian Library, Laud 636. XII/XIImed. Peterborough.

Chronique anglo-saxonne (s'achève sub anno 1154).

25. [Ker \$373] Rochester Cathedral. Textus Roffensis. XII1. Rochester.

Lois en latin et en anglais.

26. [Ker \$379] Salisbury Cathedral 150. XI/XII. Sherborne.

Psautier en latin du $\mathrm{X}^{2}$, glose anglaise du XI/XII.

27. [Ker \$398] Worcester Cathedral F.174. XIII1. Worcester.

Ælfric. Grammaire et Glossaire, Worcester Fragments, Body and Soul.

Abordons directement la question des provenances de ces manuscrits. La liste de Ker montre que celles-ci ne sont qu’imparfaitement connues et que, parmi elles, certaines sont proposées avec circonspection. Sur les vingt-sept manuscrits regroupés ici, seuls quatorze se sont vu attribuer un lieu de composition. Néanmoins, leur distribution ne semble pas être due au hasard : dix des quatorze proviennent des scriptoria du Sud-Est, quatre du Sud-Ouest. Dans le premier cas, à Londres est attribuée une copie des lois de Cnut (No 6). à Canterbury (Kent) un psautier trilingue $\left(\mathrm{N}^{\circ} 8\right)$, une chronique anglo-saxonne $\left(\mathrm{N}^{\circ} 10\right)$ ainsi que l'Évangile en deux exemplaires (Nos 19, 22); à Rochester (Kent) les sermons d'Ælfric (Nos 3, 16) et un recueil de lois $\left(\mathrm{N}^{\circ} 25\right)$, alors que Peterborough (Northamptonshire, $\mathrm{N}^{\circ} 24$ ) conserve la tradition historiographique en anglais jusqu'à l'avènement en 1154 du roi Henri II Plantagenêt. Dans le deuxième cas, deux copies des Sermones Catholici d'Ælfric sont attestées dans les Midlands occidentales (Nos 21 et 23), tandis que sa Grammaire et son Glossaire furent copiés à Worcester (Worcestershire, $\mathrm{N}^{\circ} 27$ ). Finalement, au cœur du Sud-Ouest, dans le Dorset, le scriptorium de Sherborne aurait produit un psautier glosé $\left(\mathrm{N}^{\circ} 26\right)$.

Ces preuves, en dépit de leur caractère incomplet, laissent penser que l'importance accordée aux régions des Midlands occidentales et du Sud-Ouest dans le cadre de l'activité littéraire en langue vernaculaire ne devrait pas être admise au détriment du Sud-Est du pays : les scriptoria de

17. Ladite "main" a fait l'objet d'une étude récente : Christine FravZris. The Tremulous Hand of Worcester. Oxford. 1992. 
Canterbury, de Rochester et de Londres sont aussi productifs. La volonté de préserver les réussites littéraires du passé fut donc un phénomène généralisé ${ }^{18}$.

Présents dans les deux groupes de manuscrits, partagés ici selon leur lieu de composition, sont les Sermones Catholici d'Ælfric ${ }^{19}$. indiquant que le désir de les préserver fut ressenti à travers le pays entier. Le contenu des autres manuscrits appartenant à la liste établie par Ker ne fait que souligner que ce sont ces collections de sermons. avant tout autre texte ou genre littéraire. qui ont attiré l'attention et l'assiduité des copistes, et que parmi elles c'est l'œuvre d’Ælfric qui a primé.

En tout, il existe environ vingt-cinq manuscrits des deux séries des Sermones Catholici, dont sept en date du XII ${ }^{\mathrm{s}}$ s. ${ }^{20}$. Ils sont indiqués ci-dessous avec leurs numéros de liste ainsi que les sigles manuscrits (auxquels référence sera faite ultérieurement) adoptés par les éditeurs.

- Cambridge. University Library. Ii.1.33, XII2 ( $\mathrm{N}^{\circ}$ 1. Clemoes et Godden ms. L)

- Cambridge. Corpus Christi College, 302, XI/XII (N 2. Clemoes et Godden ms. O)

- Cambridge. Corpus Christi College, 303. XII ( $\mathrm{N}^{\circ}$ 3. Clemoes et Godden ms. $C$ )

- Cambridge, Corpus Christi College, 367. XII ( ${ }^{\circ}$ 4. Clemoes et Godden ms. $f a$ )

- Londres. British Library. Cotton Faustina A.ix. XII ${ }^{1}\left(\mathrm{~N}^{\circ}\right.$ 13. Clemoes et Godden ms. $\left.N\right)$

- Londres, British Library, Cotton Vespasian D.xiv. XIImed. ( ${ }^{\circ} 16$. Clemoes et Godden ms. $\left.G\right)$

- Oxford. Bodleian Library. Bodley 343, XII2 $\left(\mathrm{N}^{\circ}\right.$ 21. Clemoes et Godden ms. B) ${ }^{21}$

Un autre manuscrit du XII ${ }^{e}$ s., Oxford, Bodleian Library, Hatton 116 ( $\mathrm{N}^{\circ} 24$. Clemoes ms. S), ne conserve que treize sermons de la première série; ceux de la deuxième série sont absents.

Le corpus de sermons anglais du XII ${ }^{\mathrm{e}}$ s. est donc constitué principalement des œuvres d'Ælfric et de Wulfstan: s'ajoutent à celles-ci les collections tardives de Lambeth (Londres, Lambeth Palace, ms 487) et de Trinity (Cambridge, Trinity College, ms. B.14.52), collections qui incarnent les techniques de composition propres au modèle indigène, mais qui ne sont pas, dans l'ensemble, le fruit d'un remaniement de textes existants ${ }^{22}$. À cause de leurs dates tardives - on pense que le manuscrit de Lambeth appartient à la toute fin du XII' s., celui de Trinity au XIII $\mathrm{s}$. - ces collections ont été rejetées par Ker lors de la constitution de sa liste, bien qu'elles furent susceptibles d'y figurer grâce à leur contenu. Également rejeté fut le ms. Bodleian Junius 1 , l'unique témoin du texte connu sous le nom de l'Ormulum, probablement à cause de son caractère linguistique : son auteur, le chanoine augustinien Orm, n'écrit pas en vieil-anglais, mais en moyen-anglais ${ }^{23}$. Bien que le rejet de ce manuscrit par Ker, qui s'appuie sur un critère strictement linguistique, soit tout à fait justifié, je m'efforcerai de démontrer qu'au point de vue proprement littéraire l'œuvre d'Orm s'avère très pertinente dans le cadre de mon enquête.

L'Ormulum, aujourd'hui un fragment, comprend une série d'homélies exégétiques en vers qui expliquent et commentent la vie du Christ comme elle est racontée dans les Évangiles, dont les versions (parfois différentes) sont harmonisées. Ces homélies sont aujourd'hui composées d'environ dix mille vers, et on estime que dans son état complet le texte aurait dépassé cent

18. À prendre en considération aussi sont les preuves rassemblées par Elaine Trinarn\&: "The Dates and Origins of Three Twelfth-Century Old English Manuscripts". dans Philip P(ilsiano et Elaine M. Trehtar.n.. éd.. Anglo-Saxon Manuscripts and their Herituge. Aldershot. 1998. p. 227-253. Son argument renforce limportance accordéc ici au Sud-Est du pavs.

19. Nin 3 et 16 . d une part. 21 et 23 . d'autre part.

20. $\mathrm{Ne}$ sont pas pris en compte les manuscrits fragmentaires dont le contenu original est difficile à établir.

21. Deux manuscrits de ce sous-groupe. les No 12 et 21 constituent également une source importante de l'exuvre de Wulfstan.

22. Voir Joseph HAl... éd.. Selections from Early Middle English. 11.30-1250. Oxford. 1920. 2 vol.. qui présente trois textes du manuscrit de Lambeth et un du manuscrit de Trinity College. (ambridge. La place des Trinity Homilies dans le débat qui entoure la notion de continuité littéraire est examinée par Jerome OrtgeN. "The Trinity college Ascension Sermon : Sources and Structure," Medicevial Studies, 45. 1983. p. 410-417.

23. Quelle que soit la nature des changements linguistiques observables entre les sermons dÆElfric et les homélies de lOrmulum. lappartenance de ce dernier aux textes "en moyen-anglais" ne détermine en rien ses prédilections littéraires. 
soixante mille vers ${ }^{24}$. L'œuvre s'inspire probablement d'un commentaire biblique, telle la Glossa Ordinaria, et elle fut composée, selon une étude récente paléographique, avant 1180 à Bourne (Lincolnshire), non loin de Peterborough ${ }^{25}$. En outre, le temps jugé nécessaire à l'achèvement de ce texte très long fait remonter les débuts de sa composition vers le milieu du XII ${ }^{\mathrm{e}} \mathrm{s}$., peut-être aux alentours de 1155/60, c'est-à-dire à l'époque où le dernier scribe dc la Peterborough Chronicle posa sa plume pour la dernière fois (en 1154) ${ }^{26}$. Ces homélies sont donc représentatives du dialecte des Midlands-Est ou des Midlands Sud-Est, et non pas des Midlands Nord-Est, comme le veut la critique traditionnelle.

La probabilité que l'Ormulum soit redevable aux commentaires de la Glossa Ordinaria sur les Évangiles. commentaires créés à Laon, dans le nord de la France, pendant les premières décennies du $\mathrm{XII}^{\mathrm{e}}$ s., révèle l'une de ses caractéristiques les plus importantes : ces homélies représentent un nouveau départ en matière d'instruction pastorale destinée en premier lieu aux prédicateurs, puis à un auditoire non ou peu instruit, et ceci, comme nous le rappellent André Crépin et Hélène Taurinya Dauby, "avant [que] les décrets du quatrième concile de Latran de 1215 impos[e]nt les sermons sur l'Évangile » ${ }^{27}$. Le fait que la forme métrique adoptée par Orm. le septénaire, soit également issue de la tradition latine, éloigne davantage son texte du modèle indigène, amplement représenté dans des manuscrits vieil-anglais cités plus haut ${ }^{28}$. Sur le plan chronologique, l'œuvrc d'Orm se trouve donc plus proche du milieu intellectuel responsable du maintien en circulation des sermons d'Ælfric que de celui qui a produit les textes du groupe de sainte Catherine plus d'un demi-siècle plus tard.

En résumé, on peut constater que, dans l'ensemble, les manuscrits anglais du XII $\mathrm{s}$. témoignent de la survie du sermon vieil-anglais au Sud, Sud-Est, aux Midlands orientales et occidentales du pays. La situation privilégiée habituellement accordée aux régions du Sud-Ouest ne repose donc pas sur un fondement solide. En plus, il est évident que la constitution d'un nouveau corpus de textes destinés à être prêchés s'effectuait en même temps que la transmission continue du corpus ælfricien, et dans les scriptoria voisins. C'est à la lumière de ces circonstances que je me propose d'aborder l'étude comparée du thème de la militia Christi, thème central dans les stratégies de composition chez Ælfric et son successeur, Orm.

L'image du soldat du Christ occupe une place non négligeable dans la littérature sermonnaire tout au long du Moyen Âge, et elle est évoquée à maintes reprises, en particulier par ceux qui constituaient les auctoritates pour les prédicateurs anglais : Augustin, Grégoirc lc Grand, Bc̀dc, Smaragde et autres, tels qu'ils apparaissent dans les homéliaires carolingiens. Mais on peut en même temps légitimement supposer que, pour des raisons autres que littéraires, la nécessité de

24. La seule édition disponible est celle de Robert HOLT, The Ormulum, with the Notes and Glossary of Dr. R.M. White, Oxford, 1878, 2 vol. (ci-après WhITE-Holt), édition jugée "médiocre" par Mossé (Manuel, voir n. 6 ci-dessus, vol. 2. p. 212). La EETS envisage la publication d'un facsimile du ms. Junius 1. Omelya est le terme employé par Orm luimême. Voir sa note ajoutée à la leçon biblique de l'homélie 201, imprimée par WuITE-Holt sous la rubrique Texts après la Dedication (partie de l'édition où les pages ne sont pas numérotées).

25. Sur les sources, la discussion la plus approfondie est celle de Heinrich C. MaTTHES. Die Einheitlichkeit des Orrmulum. Heidelberg. 1933. Voir ma discussion de certains de ses résultats: "Sources for the Ormulum : a Reexamination". Neuphilologische Mitteilungen. 84, 1983, p. 419-436 et "New Sources for the Ormulum". Neophilologus, 68. 1984. p. 444-450. La contribution majeure au débat sur la date et la provenance du manuscrit de ce texte est celle de Malcolm B. Parkes, "On the Presumed Date and Possible Origin of the Manuscript of the 'Orrmulum' : Oxford. Bodleian Library. MS Junius 1 ". dans Stanı.l:Y et Gray. éd.. Five Hundred Years of Words and Sounds (op. cit. n. 14), p. 115-127.

26. Sur la longévité supposée de lauteur, voir Joan Tt'RVILIF-Petre., "Studies on the Ormulum MS ". Journal of English and Germanic Philology. 46, 1947, p. 1-27. J. Turville-Petre ne propose aucune date précise dans son étude: la responsabilité d'avoir avancé celle de $1155 / 60$ est entièrement la mienne.

27. Crípin et Taurinya. Histoire de la littérature anglaise (op. cit. n. 11). p. 66. Sur Laon et les écoles, on consultera les travaux de Beryl Smalli:y, réunis en un volume. The Gospels in the Schools, c: 1100 - c. I280. Londres, 1985.

28. Et cela en dépit de lemploi du septénaire dans un autre sermon versifić anglais. le Poema Morale, contemporain d'Orm. Voir Hall, éd., Selections from Early Middle English, 1130-1250 (op. cit. n. 22). I. p. 30-53. Pour H.H. Gilun7. Die Literarästhetik des europäischen Mittelahters. Bochum, 1937, p. 311 et ss. l'influence exercée ici par la tradition poétique latine fut considérable. 
prêcher la vérité spirituelle de ce topos s’est fait ressentir chez Ælfric de manière incisive. Je fais allusion à la propension de certains clercs de son époque à abandonner leurs armes spirituelles en faveur de celles associées à l'activité militaire. A la fin de sa paraphrase de l'histoire des Maccabées (dans les Vies des Saints). Ælfric, défenseur de l'idéal monastique et de la vie régulière, laissant de côté ses activités de traducteur et d'adaptateur, ajoute un bref récit intitulé dans les manuscrits Qui Sunt Oratores, Laboratores, Bellatores, où il aborde le sujet des trois états. À propos des oratores, il déclare :

$\mathrm{Nu}$ se munuc be bihð to benedictes regole and forlæt ealle woruld-ðingc. hwi wile he eft gecyrran to woruldlicum wæpnum. and awurpan his gewinn wið ba ungesewenlican fynd his scyppende to teonan? Se godes peowa ne mæg mid woruld-mannum feohtan gif he on pam gastlican gefeohte ford-gang habban sceall ${ }^{29}$.

L'accusation est clairement énoncée, et d'autres témoins confirment, indépendamment, qu'elle fut bien fondée. L'entrée de la Chronique anglo-saxonne pour l'année 1016, par exemple, indique que Wulfsige, abbé de Ramsey, fut tué sur les champs de bataille à Ashington (Essex), à côté de l'évêque Eadnoð :

...xt pære dune pe man hæt Assandun... Đær ahte Cnut sige. 7 gefeaht him wid ealle Engla peode. pa wearð pær ofslægen Eadnoð biscop. 7 Wulfsie abbod. 7 Ælfric ealdorman. 7 Godwine ealdorman. 7 Ulfkytel of Eastenglan, 7 Æpelward Ælfwines sunu ealdormannes, 7 eall seo duguð of Angelcynnes peode ${ }^{30}$.

La même source raconte encore que, quarante ans après les exploits de Wulfsige, un certain évêque, Leofgar, fidèle à son nom :

forlet his crisman 7 his rode 7 his gastlican wapnu, 7 feng to his spere 7 to his swurde, and swa for to ferde ongean Griffin pone wyliscan cining. 7 he wearo pær ofslagen, 7 his preostes mid him... ${ }^{31}$.

Sans vouloir forger un lien direct entre les préoccupations de ces évêques et les stratégies littéraires du sermonnaire, il est difficile de croire qu'Elfric soit resté insensible à la contradiction flagrante manifestée par un tel comportement.

\section{L'image de la militia Christi : ses origines et sa réalisation en vieil-anglais}

Abordons maintenant la question des origines de cette image, sa transmission et sa forme littéraire en vieil-anglais ${ }^{32}$, vue principalement, mais non pas exclusivement, dans l'œuvre d'Ælfric. L'activité de la lutte spirituelle, la militia Christi, est propre au clergé, et en particulier

29. SkEat (éd. cit. n. 4). II. p. 124. Ce récit est attesté aujourd hui dans cinq manuscrits dont deux - Cambridge. University Library li.1.3.3 et Cambridge. Corpus (hristi College 303 - sont du XII' s. Une traduction partielle de ce passage fut proposée par Georges DLBY. Les trois ordres, ou limaginaire du féodalisme. Paris. 1978. p. 132 : "Le moine qui se plie à la règle bénédictine et renonce aux choses du monde. pourquoi retournerait-il aux armes terrestres. abandonnerait-il la lutte quil a entreprise contre les ennemis invisibles et mécontenterait-il son créateur " "Le serviteur de Dieu ne doit pas entrer dans le combat à côté des hommes de ce monde, siil compte réussir dans sa lutte spirituelle].

30. Cité du ms. D. éd. G. P. ClbBin. The Anglo-Saxon Chronicle : A Collaborative Edition. vol. 6. ms. D. Cambridge. 1996. p. 62 .

31. Chronique anglo-saxonne. s.a. 1056 (mss $C$ et $D$ ): la citation est tirée encore du ms. $D$ (éd. cit. p. 75). Leofgar est composé de leof (cher. aimé) et de gar (lance).

32. Une vue densemble est proposée par Joyce Hill.. "The Soldier of (hrist in Old English Prose and Poetry". Leeds Sundies in English. n.s. 12. 1981. p. 57-80. 
aux moines, considérés comme les successeurs des martyrs ${ }^{33}$. En revanche, pour celui qui est le soldat du Christ, la lutte armée est. comme l'indique clairement Ælfric, une activité proscrite. Dans une lettre adressée à Wulfstan, alors évêque de Londres, il explique le raisonnement de cette interdiction : la main responsable d'avoir fait couler le sang d'un homme n'est pas digne de bénir, sur l'autel, le calice qui contient le sang du Christ.

Ergo non potest [le prêtre] in ambabus militiis simul starc, quia illa manus que humanum sanguinem effuderit non potest digne domini calicem sanctificare ${ }^{34}$.

La Règle bénédictine caractérise la vocation monastique en termes de lutte : les cénobites sont ceux qui sont monasteriale, militans sub regula vel abbate ${ }^{35}$. Des anachorètes on apprend :

id est heremitarum, horum qui non conversationis fervore novicio, sed monasterii probatione diuturna, qui didicerunt contra diabolum multorum solacio iam docti pugnare, et bene extructi fraterna ex acie ad singularem pugnam heremi, securi iam sine consolatione alterius, cogitationum. Deo auxiliante, pugnare sufficiunt ${ }^{36}$.

Dans son prologue, Benoît s'adresse au moine (en se servant du pronom de la deuxième personne, tu, pour Ad te) qui Domino Christo uero regi militaturus, oboedientiae fortissima atque praeclara arma sumis ${ }^{37}$. Et bien que la terminologie habituellement employée - militia, miles, etc. - ait pris une signification étroite, spécialisée (celle de "service", notion présente dans l'emploi d'oboedientiae), les éléments martiaux de la métaphore ne perdent pas de leur valeur sémantique ${ }^{38}$.

L'emploi de l'image de la lutte spirituelle remonte à l'âge des apôtres. Elle s'inspire des paroles de saint Paul, surtout de celles adressées aux Éphésiens :

Induite vos armaturam Dei, ut possitis stare adversus insidias diaboli, quoniam non est nobis colluctatio adversus carnem et sanguinem, sed adversus principes, et potestates, adversum mundi rectores tenebrarum harum. contra spiritualia nequitiae, in caelestibus. Propterea accipite armaturam Dei ut possitis resistere in dic malo, et in omnibus perfecti stare. State ergo succincti lumbos vestros in veritate, et induti loricam iustitiae. et calceati pedes in praeparatione Evangelii pacis : in omnibus sumentes scutum fidei, in quo possitis omnia tela nequissimi ignea extinguere : et galeam salutis assumite : et gladium spiritus (quod est verbum Dei) per omnem orationem... [ch. 6, versets 11-18].

D'autres évocations de ce thème chez saint Paul soulignent l'importance qu'il accordait à cet aspect de son enseignement ${ }^{39}$. Ces formulations ont exercé une influence profonde sur les

33. La distinction normalement faite entre le clergé séculier et le clergé régulier ne s`applique guère à la situation en Angleterre pendant et après la période de la réforme bénédictine car, presque sans exception, les évêques furent choisis parmi les membres des communautés monastiques. À ce sujet. voir Frank BARI.ow. The English Church. 10001066: A History of the Later Anglo-Saxon Church. 2c éd., Londres. 1979. p. 311-338.

34. Elfric. lettre à Wulfstan. éd. Bernhard FeHr. Die Hirtenbriefe Alfrics, Hambourg. 1914 (Bibliothek der angelsächsischen Prosa. 9), p. 225.

35. La règle de Saint Benô̂t. éd. Adalbert de Vogứ et Jean Ni:Lfvill.E. Paris, 1971/72, 6 vol. (Sources Chrétiennes $181 / 86$ ), t. I. p. 436

36. Ibid., p. 436-438. * ... autrement dit des ermites. Ce n'est pas dans la ferveur récente de la vie religieuse, mais dans l'épreuve prolongée d’un monastère qu'ils ont appris à combattre le diable. instruits qu'ils sont désormais grâce à l'aide de plusieurs. et bien aimés dans les lignes de leurs frères pour le combat singulier du désert. ils sont désormais capables de combattre avec assurance les vices de la chair et des pensées. sans le secours d’autrui, par leur seule main et leur seul bras, avec l'aide de Dicu."

37. Ibid.. p. 412. "... abandonnant les propres volontés pour servir le Seigneur Christ, le roi véritable. prends les armes très puissantes el glorieuses de lobéissance".

38. Voir l'étude d’Eugène Manning, "La signification de Militare - Militia - Miles dans la Règle de saint Benoît ". Revue bénédictine. 72, 1962. p. 135-138.

39. E.g. Rom. 13:12:2 Cor. 10:4:1 Thess. 5:8: 1 Ioan. 5:4, etc. 
auteurs des Vies des saints fondateurs du monachisme - Paul l'ermite, de saint Jérôme ${ }^{40}$. saint Antoine, d'Athanase ${ }^{41}$. saint Martin ${ }^{42}$ - dont les œuvres constituaient une source inestimable puisée par les hagiographes anglais. On pense au moine anonyme de Whitby qui a composé une Vie de saint Grégoire le Grand ${ }^{43}$, à Bède pour sa Vie de saint Cuthbert ${ }^{44}$. et à l'œuvre de Félix sur Guthlac. le jeune guerrier qui a abandonné la lutte armée pour un combat considéré plus noble ${ }^{45}$. C'est également l'héritage des sermonnaires de langue anglaise. Chez Ælfric, les fruits de cet héritage atteignent leur apogée.

Limage du soldat du Christ repose sur un fondement militaire - loricam, galeam, gladium, et, nous l'avons vu (à la note 45). Félix, s'inspirant du modèle paulien, ajoute d'autres armes: arcus, sagitta - mais elle évoque une activité totalement étrange au monde du guerrier de ce monde. C'est la résolution de cette contradiction apparente qui révèle la force et la signification de la métaphore : les termes martiaux ne peuvent être interprétés littéralement, ils ne peuvent donc fonctionner que dans un monde spirituel, un monde où seul l'esprit (ou les esprits) existe(nt).

Lorsque l'on examine la manière dans laquelle l'image est présentée en anglais, on constate, malgré la popularité évidente de la formulation paulienne dans l'Épître aux Ephésiens, que les traductions sont rares. L'une d'elle, tirée du sermon, Dominica in Media Quadragesima : secunda sententia de hoc ipso d'Ælfric, se lit ainsi :

Ymbscrydad eow mid Godes wæpnunge. pæt ge magon standan ongean deofles syrwungum. for dan de us nis nan gecamp ongean flæsc and blod. ac togeanes deofellicum ealdrum. and gastlicum yfelnyssum: Standad eornostlice mid begyrdum lendenum on soðfæstnysse. and ymscrydde mid rihtwisnysse byrnan. and nymað pæs geleafan scyld. and ðæs hihtes helm. and pæs halgan gastes swurd. pæt is Godes word... ${ }^{46}$.

Plus souvent, le catalogue d'armes se trouve résumé dans le récit en anglais par la phrase (mid) gastlicum wapnum, ("avec des armes spirituelles") ou par une formulation similaire, comme si le fait de les nommer risquait d'embrouiller le sens essentiel. Cette volonté supposée chez Ælfric de minimiser l'impact du modèle séculier sur le fonctionnement de la métaphore est reflétée aussi dans l'importance accordée aux aspects passifs de la présentation. dans les formulations

40. Comparer (un nain horrible apparaît devant saint Antoine sur son chemin vers saint Paul) : « Nec mora, inter saxosam convallem haud grandem homunculum videt. aduncis naribus. fronte cornibus asperata. cuius extrema pars corporis in caprarum pedes desinebat. Ad hoc Antonius spectaculum. scutum fidei et loricam spei, ut bonus praliator arripuit... " Jérôme. Vita S. Pauli Primi Eremitae. P.L.. t. 23. col. 23.

41. Athanase. dans sa Vie de saint Antoine. raconte : "Ian enim senex erat. Ibi autem dum conuersatur. quantas colluctationes sustinuit, ut scriptum est, non adversus carnem et sanguinem sed adversus adversarois daemones, ab his qui (ad) illum introibant didicimus". Voir l'éd. de H. Hoppenbrotiwt:Rs, La plus ancienne version latine de la Vie de saint Antoine par saint Athanase. Nimègue. 1960, ch. 60. p. 145.

42. Voir supra note 1.

43. Rares sont les évocations de la militia Christi dans ce texte. On peut comparer cependant : "Quod vir sanctus videns consueta contra eos. iuxta apostolorum. adsumendo armatorum Dei. primum cricic Christi signaculum hostilem ocius effugavit insaniam ". Ed. Bertram Col(jRavl. The Earliest Life of Gregory the Great. Lawrence, 1968, ch. 22. p. 112.

44. Sa dette envers la formulation biblique saute aux yeux: Bède explique que Cuthbert, par un acte de foi. imite les miracles faits par les Pères de l'Église. et ajoute : "Nec mirandum perfectos et fideliter Deo seruientes uiros tantam contra uim flammarum accipere potestatem, qui cotidiana uirtutum industria et incentiua suae carnis edomare. et omnia tela nequissimi ignea norunt extinguere ".

Sur l'île de Farne. sa lutte continue : "Nullus hanc facile ante famulum Domini Cuthbertum solus ualebat inhabitare colonus. propter uidelicet demorantium ibi phantasias demonum. Verum intrante eam milite ('hristi. armato galea salutis, scuto fidei. et gladio spiritus quod est uerbum Dei. omnia tela nequissimi ignea extincta. el ipse nequissimus cum omni satellitum suorum turba porro fugatus est hostis." Voir l’éd. de Bertram Coloravl. Tivo lives of St. Cuthbert. (ambridge, 1940. p. 202 pour le premier passage. p. 214 pour le second.

45. Sa détermination dentrer dans la militia Christi est exprimée ainsi par Félix : "Deinde praecinctus spiritalibus armis adversus teterrimi hostis insidias sctum fidei. loricam spei. galeam castitatis. arcum patientiae. sagittas psalmodiae. sese in aciem firmans, arripuit". éd. Bertram Colgrave. Felix's Life of St. Guthlac. Cambridge. 1956. p. 90.

46. (GoDD):Néd. cit n. 4. p. 110-126: p. 123 pour le passage cité qui représente une version quelque peu condensée du texte biblique. 
habituelles par lesquelles les écrivains annoncent que le miles a remporté la victoire spirituelle et, en particulier, dans le choix du ou des verbes pour exprimer cette victoire.

Pour illustrer ce propos, il me faut maintenant mettre en évidence comment fut constituée verbalement l'image du soldat du Christ en vieil-anglais, telle qu'elle se trouve employée habituellement à la fois par Ælfric et par d'autres sermonnaires, anonymes, qui le précédèrent. Les manuscrits choisis pour l'établissement du texte ælfricien - Londres, British Library ms. Royal 7 c.xii pour l'édition de Clemoes, et Cambridge, University Library ms Gg.3.28 pour celle de Godden - datent de la fin du $x^{\mathrm{e}} \mathrm{s}$. Ils sont donc contemporains de leur auteur et conservent un texte très proche de ses intentions ${ }^{47}$. Pour chacun des extraits tirés des $S C$, je préciserai si une version plus tardive est conservée parmi les mss $B, C, F a, G, L, N$ et $O$, et j'indiquerai, entre parenthèses, les divergences lexicales signalées par les éditeurs, en me servant de ces mêmes sigles manuscrits ${ }^{48}$. Les extraits qui suivent ne représentent qu'une infime partie des évocations de cette métaphore dans le corpus; ils sont, néanmoins, tout à fait représentatifs des préférences lexicales et thématiques des auteurs anglais.

Tout d'abord, l'enseignement d'Ælfric sur le rôle des martyrs se trouve clairement énoncé dans le sermon pour la fête de la naissance de saint Étienne, premier de ce rang, tiré de la deuxième série des $S C$. Il est conservé dans deux manuscrits, l'un et l'autre du XI ${ }^{e} \mathbf{s}$. :

Witodlice des halga cyðere. and his xftergengan wæron gewitan pyses geleafan. and disum geleafan hi cyddon gecyðnysse oferswiðende pisne feondlican middaneard. na ongean feohtende ac sweltende (Godden, éd. cit., p. 16).

Dans le texte correspondant de la première série des SC, la Passio d'Étienne, le martyr est désigné comme apela cempa (noble guerrier), désignation qui caractérise bon nombre de telles évocations. Par exemple, saint André, emprisonné par un juge perfide, nommé Egeas, fait appel à la foule, venue à sa rescousse, pour qu'elle n'intervienne pas en sa faveur (car il a hâte d'obtenir la couronne du martyre) :

Mine gebropra ne astyrige ge pone stillan drihten to xnigre yrsunge mid eowerum anginne: Ure hxlend wæs belæwed. 7 he hæfde gepyld; he ne flat ne ne (he L) hrymde ne nan man his stemme on strætum ne gehyrde: Habbad cow on stillnysse. 7 sibbe. 7 ne hremmad minne martyrdom. ac swibor gearciad eow sylfe swa swa godes cempan. 5 ge mid unforhtum mode ealle beowracan 7 lichamlice wita purh gepylde oferswipan (Clemoes, éd. cit., p. 515) ${ }^{49}$.

Encore une fois, dans sa Passio Petri et Pauli, pour laquelle Ælfric se sert d’une version apocryphe de l'histoire de Simon le Magicien ${ }^{50}$, le Christ apparaît à saint Pierre pour l'encourager dans sa lutte contre son adversaire :

Se dry simon. 7 se wælhreowa nero sind mid deofles gaste afyllede. 7 syrwiad ongean (penced hembe B) de. ac ne beo pu afyrhte. ic beo mid be 7 ic asende minne beowan paulum pe to frofre. se stapd (gæð B) tomerien into romana byri. 7 git mid gastlicum gecampe winnað ongean pone dry. 7 hine awurpad into hellegrunde. 7 gyt syððan samod to minum rice becumað mid sige martyrdomes (Clemoes, éd. cit., p. 393).

La magie pratiquée par Simon ne lui permet pas de pénétrer la pensée secrète du saint. et l'empereur Néron annonce leur défaite ainsi : "hwæt is nu simon ic wene wyt synd oferswiðde (ofercumenc B) » (id., p. 395).

47. Sur le manuscrit de Cambridge. Godden, éd. cit., n. 4. p. XXI. dit : "This is a remarkably reliable manuscript...very carefully written and containing no apparent corruptions apart from some minor crrors of copying."

48. Les divergences purement orthographiques ou syntaxiques ne sont pas prises en considération ici.

49. Lextrait appartient à la deuxième partie du sermon. la Passio. Des manuscrits du xire s. seuls $C$ et $L$ la conservent.

50. Source identifiée par Max FóRSTrR. "Üher die Quellen von Ælfrics Exegetischen Homiliae Catholicae ". Anglia. 16. 1894. p. 1-61, surtout p. 18. 
De la même manière, dans une discussion des noms par lesquels le Christ peut être connu Sagesse, Parole. Brebis et Lion - incorporée dans le sermon pour la nativité de saint Jean Baptiste, Ælfric dit ceci au sujet du terme "lion»:

he is leo geciged (ihatæn B). of iudan mæigðe. dauides wyrtruma. for pan $ð \mathrm{e}$ he durh his godcundlican strencðe pone micclan deofol mid sige his prowunge oferswidde (ofercom B) (Clemoes, éd. cit., p. 384).

C'est précisément ce sentiment que l'on retrouve dans le corpus anonyme d'homélies anglaises, antérieur en date à celui d'Ælfric. Prenons, à titre d'exemple, cet enseignement tiré d'une collection, aujourd'hui fragmentaire, connue sous le nom des Blickling Homilies. Au sujet de la tentation du Christ (Mt. 4, 1-11: Lc. 4. 1-11), le prédicateur annonce ${ }^{51}$ :

Crist oferswipde pat deofol mid pisse cypnesse [de l'évangile]...Ac us is to smeagenne pxt Drihten on pære costunge nolde his pa myclan miht gecypan, se be mihte bone costigend instepes on helle grund besencean gif he wolde. Ac mid pon worde pæs godcundan gewrites he hine oforswipde. Mid his gepylde he us bysene onstealde...

L'enseignement chez Ælfric, pour la même fête, met en évidence le rôle rédempteur du Christ dont la victoire est assurée grâce à l'exercice de l'humilité :

Se hælend com to mancynne. for $\partial i \hbar$ he wolde ealle ure costnunga oferswiðan. mid his costnungum. 7 oferswiðan urne pone ecan deað mid his hwilwendlicum deaðe. Nu wæs he swa eadmod t he gepafode pam deofle he his fandode ${ }^{52}$ (Clemoes, éd. cit., p. 267).

Finalement. je cite un passage de la Passio Machabeorum (incorporée dans les Vies des Saints), qui est une paraphrase d'extraits tirés des deux livres des Maccabées. dans lequel Ælfric, en s'éloignant du texte biblique, interprète le comportement militaire de Judas Maccabée à la lumière de ce qui fut enseigné par le Christ dans le Nouveau Testament :

On pam dagum wæs alyfed to alecgenne his fynd ${ }^{53}$.

and swipost ða hæðenan be him hetole waron.

and se wæs godes degen pe da swiðost feaht

wið heora onwinnendan to ware heora leoda.

ac crist on his tocyme us cydde oðre dincg.

and het us healdan sibbe. and soðfæstnysse xfre.

and we sceolon winnan wid pa walhreowan fynd.

pxt synd da ungesewenlican. and pa swicolan deofla

pe willad ofslean ura sawla mid leahtrum.

wið ða we sccolon winnan mid geestlicum wapnum.

and biddan us gescyldnysse simle $x$ criste.

pæt we moton ofer-winnan ba wælhreowan leahtras.

and pæs deofles tihtinge. pæt he us derian ne mæge.

bonne bcod we godes cempan on dam gastlican gefeohte.

gif we done deofol forseop purh soðne geleafan.

and pa heafod-leahtras purh gehealtsumnysse.

and gif we godes willan mid weorcum gefremmad.

Ces extraits, entièrement représentatifs je le répète, rendent possible un certain nombre d'observations. En premier lieu, les copies de l'œuvre d'Ælfric réalisées tout au long du XII's.

51. Richard Morris, éd.. The Blickling Homilies. Londres. 1874/80 (EETS os 58, 63. 73). réimpr. en un vol.. Londres. 1967. p. 31-33.

52. Elfric propose ici une version élaborée de sa source principale, saint Grégoire : «Justum quippe erat ut sic tentationes nostras suis tentationibus vinceret. sicut mortem nostram venerat sua morte superare». tirée de l'homélie Dominica Prima in Quadragesima, P.L.. 1. 76. col. 1135.

53. SkI AT, éd. cit. n. 4. t.II, p. 112. Des copies du XIl" s. se trouvent dans les mss $C$ et $l$. 
conservent un texte remarquablement fidèle à la composition originale. Il n’y a ni réécriture, ni remaniement à proprement parler: ce que l'on voit représente plutôt une mise à jour du texte où seuls les termes susceptibles de tomber en désuétude sont remplacés par des équivalents acceptables : dans les extraits cités, ihaten pour (ge)ciegan et ofercumen pour oferswiban ${ }^{54}$. En second lieu, sur le plan thématique, la vraic nature de la victoire spirituelle est signalée de manière non ambiguë : la défaite du diable ou du mal est assurée par l'exercice de la souffrance, de la patience, de l'humilité et, l'ultime paradoxe dans le sens non figuratif, par la mort - la crucifixion ou le martyre. L'accent ainsi mis sur la nécessité de la vertu et du sacrifice se voit également reflété dans l'articulation lexicale de l'image. Ma discussion de cet aspect du sujet se limite à l'examen de deux termes-clefs.

Dans un premier temps, le miles est présenté comme un cempa; sa lutte est un gecamp. Il semblerait qu'au moins le premier de ces deux termes a gardé toute sa signification militaire. Il anime le monde héroïque habité par Beowulf, entre autres ${ }^{55}$. Il est également le terme habituellement sélectionné par le ou les traducteur(s) des Évangiles ouest-saxons lorsque mention est faite des soldats romains ${ }^{56}$. C'est pour cette raison que l'emploi du terme dans le récit religieux est très souvent marqué par la présence d'un adjectif qualificatif. Bien qu'Étienne, comme nous venons de le voir, se voie désigné comme apele cempa, où l'adjectif choisi a plutôt tendance à renforcer la qualité cxprimćc par lc nom, la combinaison plus habituellement rencontrée est celle de godes cempa ou de Cristes cempa ${ }^{57}$. Parmi ceux qui sont ainsi nommés figurent les suivants : saint Jean-Baptiste (Clemoes, éd. cit., p. 488), sainte Agnès (Skeat, éd. cit., I, p. 192), saint Alban (ibid., p. 418), saint Martin de Tours ${ }^{58}$. les compagnons de sainte Cécile (ibid., II, p. 370), et saint Guthlac ${ }^{59}$. Ce trait de composition, aussi évident qu'il soit, illustre bien la perspective adoptée par des sermonnaires dans la constitution de cet aspect de leur enseignement : la force sémantique de terminologie susceptible d'évoquer un sens non voulu est soigneusement dirigée vers sa réalisation métaphorique ${ }^{60}$.

54. Les preuves textuelles proposées ici sont naturellement insuffisantes. car les passages cités servent essentiellement à révéler les termes lexicaux habituellement associés à l'image du miles. Pour renforcer la validité de ce point. je propose donc un échantillon plus important de substitutions effectuées par les scribes. Ces exemples sont tirés de la deuxième série des SC. suivis des substitutions faites par le scribe du ms. B (Bodley 343) : Godden 275/103 : ealra gereord / alle pe space : $23 / 143:$ alc gereord/ ealle spece: $54 / 70$ et $264 / 132:$ gecigede / icwadene : $254 / 160:$ gereccan / tellæn : $255 / 1$. 5 : rehton / sædon: 224/98 : bearn / sunu : 265/161 : bearna / childrene: $238 / 110$ : wyrtruma / rote: $257 / 77:$ hawad/ locæd: 228/231 : reafes / clapes: 228/232 : fnædu / scryd : 228/236 : fnædu / hem : 265/162 : untrumnys / sæcnesse : 265/157: gewinne / fahte: 263/108 : dearfleas / ncodeles:24/162.168: ateowed/iscewod. De telles substitutions sont beaucoup plus fréquentes dans ce manuscrit que dans les autres témoins du XIIc s. examinés ici : mss $L, O, C, f^{a}, N$ et $G$. Sur le plan chronologique, ces interventions scribales coïncident avec la rédaction dun nouveau corpus sermonnaire que représente l'Ormulum.

55. Friedrich KL,FBER, éd., Beowulf and the Fight at Finnsburg, 3c éd. augm., Boston. 1950. v. 1312. 1551. 1585.1948. 2044, etc.

56. À neuf reprises sur douze : Mt. 27:27: Mc 15:16: Lc. 3:14, 7:8, 23:36: Jn 19:23, 24, 32. 34. Dans les trois autres cas (Mt. 8:9. 28:12 et Jn 19:2), c'est le va. pegen qui traduit le terme latin. L'Évangile en vieil-anglais est disponible dans Roy LIUZZA. éd. The Old English Version of the Gospels, Oxford, 1994 (EETS 304). Sur la force sémantique plus restreinte de la forme gecamp, voir Joyce Hil... "On the Semantics of Old English cempa and campian ". Neophilologus. 67. 1983 , p. $273-276$.

57. Un exemple tiré de la poésie vieil-anglaise est examiné dans mon article, "OE cempa in Cynewulf's Juliana and the Figure of the Miles Christi ". English Language Notes, 17. 1979. p. 81-84.

58. La phrase célèbre de saint Martin. citée au début de cet article du texte de Sulpice Sévère a été rendue par Ælfric ainsi : "ic eom godes cempa : ne mot ic na feohtan" (SKLAT.éd. cit. n. 4. t. II. p. 226).

59. Félix se sert de la phrase Christi mil/es/ traduite dans la version anonyme en prose par Cristes cemp/al. Voir l’éd. de Paul Gonst:R. "Das angelsächsische Prosa-Leben des Heiligen Guthlaces". Anglistische Forschungen. 37.1909. p. $100-173$, surtout p. 119.

60. Dautres termes peuvent être cités. Ainsi. le va. wiga (guerrier). rencontré fréquemment dans des contextes de la lutte armée n'est que très rarement employé par les sermonnaires. Il en est de même pour le verbe scyldan (protéger). qui a une fonction métaphorique. alors que les termes neutres. de connotations ouvertement martiales. tel werian, ne l'ont pas. 
Le deuxième terme sur lequel je voudrais attirer l'attention est le verbe habituellement employé pour annoncer que la victoire spirituelle a été remportée : oferswiban. Avec presque cinq cents occurrences, selon la Microfiche Concordance de l'Université de Toronto, ce verbe devance considérablement ofercuman, oferwinnan, oferdrifan et oferfeohtan, qui sont également les termes sélectionnés pour exprimer cette même notion ${ }^{61}$. Tous ces verbes en ofer- possèdent un deuxième trait en commun : ils sont réservés, presque exclusivement, aux évocations de victoire spirituelle: ils ne figurent pas dans des descriptions de la lutte armée. telles qu'on les trouve dans la Chronique anglo-saxonne, par exemple ${ }^{62}$. Oferswipan contribue donc à la création pour le miles Christi d'une identité lexicale particulière et distincte de celle de son homologue séculier. Le choix de terminologie effectué par les auteurs vieil-anglais renforce le fondement thématique de victoire par la souffrance, par la passivité, et révèle la signification essentielle des paroles de saint Martin de Tours avec lesquelles j’ai commencé cette étude.

L’instruction religieuse æelfricienne. qui a fait autorité, fut soigneusement conservée et répétée tout au long du XII ${ }^{\leftarrow}$ s., comme en témoignent nos manuscrits. Or, il est évident, à la lumière des dates attribuées par les paléographes à ces manuscrits, que la préservation fidèle de cet enseignement traditionnel s'est effectuée précisément au moment de la composition et de la rédaction des homélies en vers qui portent le nom de leur auteur. Orm. Bien qu'il n'existe aucune preuve certaine d'une familiarité quelconque chez Orm avec le corpus de sermons vieilanglais. il serait inconcevable de supposer que ce dernier. contribuant à un genre littéraire stable. populaire dans le sens propre du terme. et bien répandu. n'avait pas pris connaissance de l'œuvre de ses prédécesseurs ${ }^{63}$. C'est dans cette perspective que je me propose de comparer la présentation de la militia Christi dans l'Ormulum avec le modèle vieil-anglais, esquissé plus haut.

\section{La synthèse littéraire de l'image chez Orm}

La conception de la vie chrétienne comme lutte spirituelle. dont les attributs thématiques et lexicaux sont stables et ordonnés, est exprimée à maintes reprises dans l'Ormulum. Tout comme les auteurs vieil-anglais, Orm s'inspire de la synthèse paulienne de l'Épître aux Éphésiens, comme l'indiquent les énoncés suivants. Premièrement. au sujet des vertus de la foi. de l'espérance et de la charité, Orm dit :

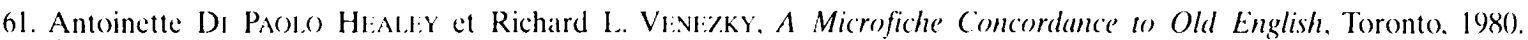
62. $\dot{A}$ une exception près : oferswipan est employé une seule fois tout au début de la version la plus ancienne de ce texte. où il traduit le latin vincere. Voir Janet BATEIY, éd., The Anglo-Saxon Chronicle : A Collaborative Edition. t. 3 : MS A. Cambridge. 1986. Les choix lexicaux faits par les traducteurs travaillant sous l'égide du roi Aelfred pendant le dernière décennie du 1 xe s. témoignent d’une certaine incohérence. Un exemple doit suffir: au livre 7. chapitre 34 de la Historiarum adiersum Paganos. Orose nous informe que Gratien. nommé empereur. s'associe à Théodose pour former un bloc militaire tres puissant : "... feng Gratianus to Romana onwalde 7 hiene hafde vi ger. 7 gesette Theodosius him to fultume. for pon him gepuhte pat pa peoda be hiora widerwinnan waron waren to swide gestrongade pat hie mon leng ne mehte mid gefeohtum oferswipan". (omme lindique léditrice de la version vieil-anglaise. la deuxième partie de cette phrase correspond a "cum adflictum ac paene conlapsum reipublicae statum uideret". phrase qui est interprétée plutôt que traduite littéralement. Pour le traducteur anonyme il n'y a. apparemment. rien d'incongru dans l'association de fecaht et oferswipan: pour Flfric un siecle plus tard. cest toui le contraire : saint Élienne entre dans le rang des martyrs. "oferswidende pisne feondlican middaneard. na ongean feohtende ac swehtende" ((ion)tis. éd. cit. n. 4. p. 16). Le texte vieil-anglais do Orose est édité par Janet Batris. The Old English Orosius. Londres. 1980) (FFTS ss 6): lextrait examiné ici se trouve à la page 153.

6.3. La présence de la rhétorique homilétique vieil-anglaise dans l'auvre d’(Orm na rien de surprenant. Voir mon étude préliminaire. "Orm's English Sources". Archiv fiur das Studium der neuceren Sprachen und Literaturen. 221.1984. p. $54-64$. 
... wha sitt iss patt wapnedd iss Wipp pise prinne mahhtess

...he maz3 stanndenn wel onnzan pe defell wipp swille wapenn $(2781-86)^{\text {th }}$

Dans le même esprit, il souligne ainsi le rôle protecteur des anges envoyés à l'homme par Dieu :

Itt tacnepp he senndepp hemm Inn till piss pesterrnesse

To frofrenn ba wakenn wel Onnzæn lape gastess

Annd stanndenn inn to shildenn hemm Fra defless swikedomess (3790-95)

Les éléments constituants de ces extraits rappellent d'emblée l'image biblique : wapnedd pour armaturam Dei (Ep. $6: 11,13)$; stanndenn onnzan pour resistere, stare $(6: 13)$; wakenn wel pour vigilantes (6:18) ; shildenn pour scutum (Dei) (6:16) et swikedomess pour insidias (diaboli) (6:11). Mais les similitudes entre les deux interprétations anglaises de l'image s'arrêtent là. Tandis qu'Ælfric et les auteurs anonymes vieil-anglais avaient tendance à minimiser l'impact du modèle séculier, Orm cherche à le rehausser. Au lieu de phrases telles que mid gastlicum wapnum ${ }^{65}$, qui résument le catalogue d'armes de saint Paul, Orm met l'accent sur la wapenn god annd stranng (4556), ou sur le fait que Job, drihhtiness kemmpe (4832), mènc sa luttc avec succès car il est wapnedd wel $(4768,4806)$ et wapnedd full wel (4787). Dans un autre endroit. Orm enseigne que l'exercice de toutes les vertus doit être effectué par la patience :

Forr pild birrp ben wipp whillc mahht To beldenn itt annd strenngenn

Swa patt itt muzhe ben till uss God wapenn $3 x n$ pe defell (2613-66).

Encore plus notable est la représentation du Christ, lors de sa tentation dans le désert, comme guerrier confronté à une épreuve de force. Orm explique pourquoi le Christ a engagé le combat avec son adversaire sans l'aide des ses anges :

patt Godess enngless nærenn nohht Abutenn ure Laferrd

Inn all patt time patt he wass Inn orresst $3 æ n$ pe defell.

patt wass, all alls hiss wille wass. Forr patt he wollde shæwenn,

patt himm nass rihht nan ned till hemm To fihhtenn $3 æ n$ pe defell,

Forr patt he mihhte himm sellf inoh Wipputenn enngless hellpe

All pwerrt ut oferrcumenn himm. To brinngenn himm to grunde,

Annd bindenn himm. annd lesenn ut Mannkinn off hise bandess.

(12534-549)

Dans ce dernier morceau, la signification des choix lexicaux effectués par le prédicateur est révélée en le comparant avec le passage suivant, tirée de la Chronique de Peterborough pour l'an 1096 ; le chroniqueur parle de l'animosité entre un certain Geoffroi Bainard et Guillaume, comte d’Eu, apparenté avec le roi du même nom :

pær (à Salisbury) beteah Gosfrei Bainard Willelm of Ou, pes cynges mæg, pet he heafde gebeon on pas cynges swicdome. and hit him on gefeaht 7 hine on orreste ofercom ${ }^{60}$.

64. Une discussion compréhensive des traits distinctifs de son orthographe est proposée par Kenneth SisaM. Studies in the History of Old English Literature. Oxford. 1953, p. 188 et ss. Le texte de l'Ormulum est. mutatis mutandis. celui de l'édition de Wurtr:-Hol:T (voir n. 24). Dans mes extraits. la dipthongue /eo/ est réduite à la voyelle /e/, conformément aux intentions d'Orm. Sur ce point. voir les quelques remarques formulées dans mon article "Orm`s English Sources" (voir n. 6.3). p. 54

65. À noter également lemploi de mid Godes waepnunge (GODdEN, éd. cit. n. 4, p. 123/466 : - SKLAT, éd. cit. n. 4. p. 416-450. etc.).

66. (ecily ('ARR. éd.. The Peterhorough Chronicle. 2c éd.. Oxford. 1970, p. 24. 
Contrairement à ce que l'on trouve chez Ælfric et les auteurs vieil-anglais anonymes, le vocabulaire associé à la lutte armée anime également celle de l'esprit. D'autres éléments de composition habituellement utilisés confirment cette nouvelle tendance. Parmi eux figure, par exemple, l'association à size (victoire) du terme oferrhannd (supériorité) dans la phrase winnenn size annd oferrhannd ou oferrhannd annd size (5460, 11421, 11481, 16965), terme qui, dans la littérature en date de la fin du XII ${ }^{\mathrm{C}}$ s., ne fonctionne que dans des descriptions de guerre armée $^{67}$.

Mais l'illustration la plus incisive de la volonté chez Orm de privilégier le caractère pugnace de l'image du miles est fournie par sa décision d'intégrer un élément tout nouveau dans la configuration traditionnelle : l'image de l'homme juste qui foule les bêtes aux pieds, tirée du verset 13 du psaume 90 (de la Vulgate). En effet, Orm associe à son autorité biblique traditionnelle une deuxième auctoritas biblique :

Super aspidem et basiliscum ambulabis

Et conculcabis leonem et draconem

La nouvelle synthèse se trouve dans de nombreux contextes. De la Vierge Marie. par exemple, il est dit que :

...3ho wass full off strenncpe annd mahht To stanndenn $3 æ n$ pe defell

Forr 3 ho tradd defell unnderrfot pwerrt ut onn alle wise (2559-62).

De plus, le Christ, dans sa tentation, remporte la victoire (tout comme l'interprétation faite par le compilateur de la Blickling Homily pour la même occasion) par l'exercice de sa skill, annd nohht / Wipp nan unnride strenncpe (12526-5237); et pourtant, cette victoire est annoncée de la manière suivante :

pe defell comm to wundenn Crist purrh grediznessess wapenn

Annd purrh patt tatt te laferrd Crist Wippstod onnzan hiss wille

pærpurrh pe laferrd oferrcomm Annd oferrtrad te defell (12496-505).

formulation qui est répétée pour chacune des trois tentations.

Encore une fois, l'opposition entre les vices et les vertus est présentée de la même façon. Par exemple, de la douceur (le fait d'être milde annd mec), Orm déclare :

piss mahht tredepp unnderrfot All grimmelez3c annd brapbe

Annd hete annd nip annd apperrmod Itt drifepp fra pin herrte $(4718-21)^{68}$.

La dernière, et peut-être la plus remarquable, illustration de ce trait de composition est celle qui présente le sacrifice de la crucifixion comme un exploit sur un champ de bataille :

Annd ure laferrd Iesu Crist Iss king off alle kingess

To fihhtenn forr hiss hall 3 he follc $\mathrm{O}$ rode $3 æ n$ pe defell

Annd mahhtiz king annd stranng inoh Inn hiss godcunnde kinde

To tredenn all unnderr hiss fot pe lape gastess strennche (18128-35).

67. Voir le Brut de Lawaman. éd. par G.L. BRoOK et R.F. LESIIF, Layamon's Brut. Londres. 196.3/78 (EETS 250) et 277). 2 vol.. v. 646-8, 1242. 9144-45.

68. Léd. de Whitk-Holt donne, par erreur, anwermod pour appermod. Sur ce dernier terme. voir Robert W. Btr(HFll:L.D. "Two Misreadings from the Ormulum MS". Medium Aium. 21. 1952. p. 37-39. 
C'est la reconnaissance de l'incompatibilité sur le plan littéral de ce nouvel élément avec son contexte, la crucifixion, qui assure l'efficacité de la métaphore.

L'Ormulum témoigne donc, pour la première fois en anglais. de l'intégration ordonnée de ce détail dans la présentation traditionnelle du topos de la militia Christi : on cherche, en vain, un tel trait de composition employé régulièrement dans le corpus vieil-anglais. En revanche, dans les récits religieux en prose datant des débuts du XIII $s$. (auxquels j'ai déjà fait allusion), la stratégie littéraire d'Orm trouve son écho. La quatrième partie de l'Ancrene Wisse (v. 1225) est un exposé sur le péril de la tentation liée, naturellement, aux sept péchés capitaux. Vers la fin de cette partie, le maître instruit la jeune anachorète ainsi :

3ef pu purh pi 3 emeles werest te earst wacliche, ant...ah art ibroht se ouerford $\bar{t}$ tu ne maht pis scheld halden o bin heorte ne wrenchen hire per under frommard be deofles earowen, nim pe aleast forð sein Beneites salue...totred te neddre heaued $\mathrm{p}$ is pe biginnung of his fondunge...Alswe, leoue suster, sone se pu eauer felest $\hbar$ tin heorte wið luue falle to eani ping eawt ouer mete, ananrihtes beo war of be neddre atter ant totred his heaued ${ }^{69}$.

Le pouvoir de fouler aux pieds ses ennemis se voit également attribué au diable, comme indique l'auteur anonyme d'un dialogue intitulé (par l'éditeur) Vices and Virtues, daté du tout début du XIII' s. : Werize se ðe wile! All ðare hwile ðe ðu art on ðese earme liue, ðu art on muchele ifihte. ba de noht ne fihten, he bied al te-treden under dieules fiet ${ }^{70}$.

S'ajoutent à ces synthèses littéraires d'importants parallèles iconographiques dans le contexte du péché et de la tentation. Tout d'abord, l'on trouve sur les fonts baptismaux de Stanton Fitzwarren (Wiltshire) [env. 1180], une représentation des huit vertus, accompagnées de l'Église (Ecclesia) et d'un chérubin, qui foulent les vices aux pieds. De la même école provient un coffret en cuivre, conservé actuellement dans la cathédrale de Troyes (Aube), qui reproduit de manière très violente le même schéma que les fonts baptismaux ${ }^{71}$. Également révélateur est un diptyque anglais en ivoire, datant de la deuxième moitié du XII ${ }^{\mathrm{e}}$ s., conservé aujourd'hui dans le Museo Nazionale à Florence. La tablette de droite est une représentation du Christ qui foule le lion (percé par une lance, aujourd'hui manquante) et le serpent aux pieds. L'inscription qui entoure cet ensemble annonce que c'est par sa mort que le Christ remporte la victoire : Rex Deus est et Homo, quem simplex signat imago quo mors est strata serpente leone notata. La tablette de gauche est composée de la figure de saint Michel qui, à son tour, foule le diable aux pieds : son inscription associe clairement l'image de la synthèse paulienne au verset du psaume $90: S v b$ fidei scvto Micahel stans corpore tvto hostem, et sternit pede, calcat cvspide pungit ${ }^{72}$.

Comment doit-on interpréter ces similitudes et ces divergences à la fois thématiques et verbales ? Premièrement, il semblerait que, pour la littérature sermonnaire anglaise des $\mathrm{X}^{\mathrm{c}}, \mathrm{XI}^{\mathrm{e}}$ et $\mathrm{XII}^{\mathrm{c}} \mathrm{s}$., la synthèse verbale chez Orm, sur laquelle repose l'interprétation de la lutte spirituelle, constitue un nouveau départ. La signification théologique reste, certes, inchangée, mais les éléments constituants de sa réalisation littéraire la rapproche de son homologue séculier, un rapprochement si soigneusement évité par les compilateurs vieil-anglais. De plus, l'accent ainsi mis sur le caractère belliqueux du miles se voit reflété dans la composition de certaines réalisations artistiques contemporaines : l'interprétation privilégiée par Orm n'est pas un

69. J.R.R. TOL.KIIN, éd., Ancrene Wisse. Londres, 1962 (EETS 249), p. 152-153. La référence au serpent renvoie également au chapitre 3. verset 15 du livre de la Genèse : "Ipsa conteret caput tuum. Et tu insidiaberis calcaneo cius".

70. Friedrich Holthatsis, éd., Vices and Virtues : Being a Soul's Confession of its Sins with Reason's Description of the Virtues. Londres. 1888-1921 (EETS os 89.159). p. 89.

71. Sur le premier. voir George Zarnicki. Later English Romanesque Sculpure, 1140-1210. Londres, 1953, p. 61 et pl. 97. Sur le coffret. voir Hans SwAR7rsiski. Monuments of Romanesque Art. 2c éd.. Londres, 1974, p. 77 et pl. 197 : English Romanesque Art. 1066-1200 [catalogue d'exposition]. Londres, 1984, p. 267-268.

72. Sur ke diptyque. voir John Brokwith. Ivory Carings in Early Medieral England, Londres, 1972. p. 136. pl. 85. 86. 
phénomène isolé ${ }^{73}$. On remarque également que les parallèles littéraires les plus proches de l'Ormulum se trouvent développés dans la prose de la fin du XII' $\mathrm{s}$. et celle des débuts du XIII - le Brut, l'Ancrene Wisse. Les nouvelles pratiques littéraires témoignées par ces derniers sont en effet anticipées par Orm plus d'un demi-siècle avant leur apparition. À la lumière de ces preuves, il serait difficile de ne pas admettre que l'extrême fidélité accordée au modèle ælfricien, témoignée par l'activité des scribes tout au long du XII s. reflète une tendance conservatrice en matière de composition littéraire de plus en plus coupée des besoins des prédicateurs de cette fin de siècle. Pour la critique actuelle, le phénomène de continuité littéraire des traditions vieilanglaises persiste jusqu'à la deuxième ou la troisième décennie du XIII ${ }^{\mathrm{c}} \mathrm{s}$.: pour ceux qui prennent en compte le témoignage de l'Ormulum, le point de coupure et de renouvellement serait plutôt placé aux alentours de 1150/60. Une telle conclusion devrait permettre de voir l'activité scribale en Angleterre au $\mathrm{XII}^{\mathrm{e}} \mathrm{s}$. sous un angle plus large que celui rencontré habituellement.

\author{
Stephen MORRISON \\ Centre d'Études Supérieures \\ de Civilisation Médiévale \\ 24, rue de la Chaîne BP 603 \\ F-86022 PoITIERs Cedex
}

73. Contrairement à ce que pense Nicholas JACOBS qui caractérise l'eeuvre comme "freak and fossil ". I'Ormulum n’est ni l'un ni l'autre, mais est tout à fait représentatif de son temps. Voir le compte rendu d’Eric G. STANIIY et D. GraY, éd. Five Hundred Years of Words and Sounds. Woodbridge. 1983, Medium Aivum. 55. 1986. p. 273-274. 